\title{
BUILDING MIXED HODGE STRUCTURES
}

\author{
DONU ARAPURA
}

This article is aimed at people who already know what mixed Hodge structures are and what they are good for, but who are not sure how to construct them. Even if one wants to treat mixed Hodge theory primarily as a black box, there will be times when one needs to open it up, look inside, and perhaps reassemble it in a different way. I would like to discuss some of the mechanics involved along the lines of Deligne's original construction [D2]. I should mention that a somewhat different and far reaching approach to constructing mixed Hodge structures has been given by M. Saito [Sa3]; I will say a few words about it at the end. However, I believe that there is still some value in this old-fashioned approach. Some of the techniques involved are quite useful in other situations, especially descent theory, which has a number of applications beyond those discussed here (e.g. [B], [GS, [GNPP], [GN], [Ha], N| $)$.

The first basic principle of the construction is that in order to get mixed Hodge structures on cohomology of a space, the necessary filtrations should be defined on the level of complexes that give rise to the cohomology. Appropriate homological conditions need to be imposed in order to get things to work. In this article, these conditions will be embodied in the notion of a $\mathbb{C}$-Hodge complex which is a fairly minimalistic reworking of Deligne's original axioms for a cohomological mixed Hodge complex. $\mathbb{C}$-Hodge complexes need not have any underlying integral or real structure, but they do possess the necessary structure to carry out the key degeneration of spectral sequence arguments. Once these technical results are established, it is fairly straightforward to impose integral structures on the $\mathbb{C}$-Hodge complexes so as to obtain mixed Hodge structures on cohomology.

The second basic principle is that in order to construct mixed Hodge structures on the cohomology of a whole class of spaces and coefficient sheaves, it is only necessary to construct explicit Hodge complexes on a subset of these pairs, provided that the general pairs can be "approximated" by the special ones. This reduction is ultimately handled by cohomological descent. However, in order to clarify the structure of the argument, the condition for guaranteeing the existence of approximations, called density, will be introduced first; the actual descent theory will be done afterwards. Verification of density in practice requires resolution of singularities.

The last section contains a rather elaborate illustration of these ideas in construction of mixed Hodge structures on the cohomology of certain constructible sheaves.

My heartfelt thanks goes out to the referees for their remarkable thoroughness. I have learned after the fact, that one of them was Steve Zucker.

Partially supported by NSF. 


\section{First Attempt}

Let me begin by trying to make a bare hands construction of a functorial mixed Hodge structure on the cohomology of a singular projective variety. This attempt will fail, but it will be instructive nevertheless.

For the record, I should recall what a mixed Hodge structure is. I will use the standard convention that filtrations indexed by superscripts are decreasing while those with subscripts are increasing. These can be interchanged by the rule $W^{-n}=$ $W_{n}$, and we do this without further comment. A mixed Hodge structure is usually taken to be a finitely generated Abelian group $H_{\mathbb{Z}}$, along with an ascending filtration $W_{\bullet}$ on $H_{\mathbb{Q}}=H_{\mathbb{Z}} \otimes \mathbb{Q}$, and a decreasing filtration $F^{\bullet}$ on $H=H_{\mathbb{Z}} \otimes \mathbb{C}$ subject to certain axioms [D2]. After jettisoning $H_{\mathbb{Z}}$ and $H_{\mathbb{Q}}$, one arrives at the notion of the $\mathbb{C}$-mixed Hodge structure (or simply just a $\mathbb{C}$-Hodge structure) which is a finite dimensional vector space $H$ together with three filtrations $\left(F^{\bullet}, \widehat{F}^{\bullet}, W_{\bullet}\right)$ which are opposed in the sense of Deligne [D2], i.e.,

$$
G r_{n}^{W} H=F^{p} G r_{n}^{W} H \oplus \widehat{F}^{q} G r_{n}^{W} H
$$

whenever $p+q=n$, where $G r_{n}^{W} H=W_{n} / W_{n-1}$. By tradition $W$ is taken to be increasing, but it will be frequently convenient to regard a $\mathbb{C}$-Hodge structure as given by three decreasing filtrations $\left(F^{\bullet}, \widehat{F}^{\bullet}, W^{\bullet}\right)$. The third filtration $\widehat{F}^{\bullet}$ plays the role of the conjugate filtration to $F^{\bullet}$. Morphisms of $\mathbb{C}$-Hodge structures are simply linear maps preserving the filtrations. In the presence of several $\mathbb{C}$-Hodge structures $H, H^{\prime} \ldots$, I will indicate the corresponding filtrations by $F^{\bullet} H, F^{\bullet} H^{\prime}, \ldots$. $\mathbb{C}$-Hodge structures possess most of the homological properties of mixed Hodge structures. For example the category of $\mathbb{C}$-Hodge structures is Abelian, and the functors $G r_{F}, G r_{\hat{F}}, G r_{W}$ are exact [D2, 1.2.5]. A $\mathbb{C}$-Hodge structure is pure of weight $n$ if $G r_{n}^{W} H=H$. The filtration $W$ will usually be suppressed for pure Hodge structures. There are no nonzero morphisms between pure $\mathbb{C}$-Hodge structures of different weights. An integral lattice on a $\mathbb{C}$-Hodge structure $\left(H, F^{\bullet}, \widehat{F}^{\bullet}, W_{\bullet}\right)$ is a finitely generated Abelian group $H_{\mathbb{Z}}$ with an isomorphism $H_{\mathbb{Z}} \otimes \mathbb{C} \cong H$ such that $W_{\bullet}$ is rational, that is spanned by $W_{\bullet} \cap\left(H_{\mathbb{Z}} \otimes \mathbb{Q}\right)$, and $\widehat{F}^{\bullet}=\bar{F}^{\bullet}$ the conjugate of $F$ with respect to the induced real structure on $H$. A mixed Hodge structure in the usual sense is the same thing as a $\mathbb{C}$-Hodge structure together with a lattice. A morphism of mixed Hodge structures is simply a morphism of $\mathbb{C}$-Hodge structures and a compatible map of lattices.

I will generally use the term complex variety to mean a reduced separated scheme of finite type over $\mathbb{C}$ with its classical topology. Suppose that $X$ is complex proper variety. By Hironaka's theorem [Hi], there exists a commutative diagram

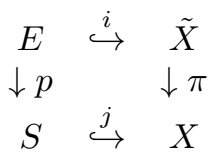

where $\pi$ is a resolution of singularities with exceptional divisor $E$, and $S=\pi(E)$. Then

Proposition 1.1. For any sheaf of Abelian groups $\mathcal{F}$ on $X$, the "Mayer-Vietoris" sequence

$$
\ldots H^{i}(X, \mathcal{F}) \stackrel{\pi^{*}+i^{*}}{\longrightarrow} H^{i}\left(\tilde{X}, \pi^{*} F\right) \oplus H^{i}\left(S, j^{*} \mathcal{F}\right) \stackrel{i^{*}-p^{*}}{\longrightarrow} H^{i}\left(E,(\pi \circ i)^{*} \mathcal{F}\right) \rightarrow \ldots
$$


is exact.

Although this fact is well known, I will give the proof since it foreshadows the results of section 5 .

Proof. I will use some basic notions from derived categories \V for succinctness, but the argument can be rewritten without them.

Let $g=\pi \circ i$. It is enough to show that the sequence

$$
\mathcal{F} \rightarrow i_{*} \mathcal{F} \oplus \mathbb{R} \pi_{*} \mathcal{F} \rightarrow \mathbb{R} g_{*} \mathcal{F}
$$

is part of a distinguished triangle. This can be checked at the stalks. If $x \notin S$, then the stalk of the sequence at $x$ reduces to

$$
\mathcal{F}_{x} \stackrel{\bar{\Xi}}{\rightarrow} \oplus \mathcal{F}_{x} \rightarrow 0
$$

which is certainly part of a distinguished triangle. If $x \in S$, let $E_{x}$ denote the fiber $g^{-1}(x)=\pi^{-1}(x)$. Then the stalk of the sequence decomposes as sum of sequences

$$
\mathcal{F}_{x} \stackrel{\bar{\rightrightarrows}}{\rightarrow} \mathcal{F}_{x} \rightarrow 0
$$

and

$$
0 \rightarrow \mathbb{R} \Gamma\left(E_{x},\left.\mathcal{F}\right|_{E_{x}}\right) \stackrel{\equiv}{\rightarrow} \mathbb{R} \Gamma\left(E_{x},\left.\mathcal{F}\right|_{E_{x}}\right)
$$

which both extend to distinguished triangles.

The proposition suggests a plan for putting a mixed Hodge structure on $H^{*}(X, \mathbb{Z})$. First use classical Hodge theory to put pure Hodge structures on the cohomology of smooth projective varieties. Then assume by induction that the cohomology of all proper varieties of dimension less than $\operatorname{dim} X$ has been equipped with a mixed Hodge structure. Choose a diagram as above, and try to impose a mixed Hodge structure on it so that the Mayer-Vietoris sequence is an exact sequence of mixed Hodge structures. Well that's the idea. The problem is that the category of mixed Hodge structures has nontrivial extensions, so compatibility with Mayer-Vietoris will not uniquely determine the mixed Hodge structure on $H^{*}(X)$. Even if one worked in the category of $\mathbb{C}$-mixed Hodge structures, which is semisimple, one would only be able to determine the $\mathbb{C}$-mixed Hodge structure on $H^{*}(X)$ up to noncanonical isomorphism. To circumvent these problems, it will be necessary to work on the level of complexes. Once this is done, it is possible to fix up this inductive approach. However it will turn out to be simpler to do the construction in one step using descent theory (following Deligne's idea). An elementary construction along lines similar to the above sketch has been given by El Zein [E]. A rather different construction has been given by Saito Sa1, Sa3].

\section{2. $\mathbb{C}$-Hodge Complexes}

Recall that a filtration on a complex $A^{\bullet}$ is a sequence of filtrations $F^{\bullet} A^{n} \subset A^{n}$ compatible with the differentials. The filtration is biregular if for each $n$, there exist $a$ and $b$ such that $F^{a} A^{n}=A^{n}$ and $F^{b} A^{n}=0$. In the sequel, I will try to conserve notation by writing $F^{\bullet}$ or simply $F$ instead of $F^{\bullet} A^{\bullet}$ when no confusion is likely.

The basic device for constructing $\mathbb{C}$-Hodge structures is a $\mathbb{C}$-Hodge complex [A] It is a biregularly trifiltered complex of $\mathbb{C}$-vector spaces $\left(A^{\bullet}, F^{\bullet} A^{\bullet}, \widehat{F}^{\bullet} A^{\bullet}, W^{\bullet} A^{\bullet}\right)$ bounded below and satisfying:

HC1. $A^{\bullet}$ has finite dimensional cohomology. 
HC2. For each $p$, the filtrations on $G r_{W}^{p} A$ induced by $F^{\bullet}$ and $\widehat{F}^{\bullet}$ are strictly preserved by the differentials (i.e. $d F^{p}=\operatorname{image}(d) \cap F^{p}$ for all $p$, and likewise for $\widehat{F}^{\bullet}$ ).

HC3. $H^{i}\left(G r_{W}^{p} A\right)$, when equipped with the filtrations

$$
\begin{gathered}
\operatorname{image}\left[H^{i}\left(F^{\bullet} G r_{W}^{p} A\right) \rightarrow H^{i}\left(G r_{W}^{p} A\right)\right] \\
\operatorname{image}\left[H^{i}\left(\widehat{F}^{\bullet} G r_{W}^{p} A\right) \rightarrow H^{i}\left(G r_{W}^{p} A\right)\right],
\end{gathered}
$$

becomes a pure $\mathbb{C}$-Hodge structure of weight $i-p$ for all $i, p$.

This is related to Deligne's notion of a mixed Hodge complex [D2; a more detailed comparison will be given below.

Example 2.1. Consider a complex $A^{\bullet}$ of $\mathbb{C}$-mixed Hodge structures with its filtrations $F^{\bullet}, \widehat{F}^{\bullet}, W^{\bullet}$. As it stands, this is not a $\mathbb{C}$-Hodge complex. But if one defines

$$
\tilde{W}^{i} A^{n}=W^{i-n} A^{n}
$$

then $\left(A^{\bullet}, F^{\bullet}, \widehat{F}^{\bullet}, \tilde{W}^{\bullet}\right)$ is.

Example 2.2. Given a $\mathbb{C}$-Hodge complex $\left(A^{\bullet}, F^{\bullet}, \widehat{F}^{\bullet}, W^{\bullet}\right)$, let $\left(A[i]^{\bullet}, d_{A[i]}\right)=\left(A^{\bullet+i},(-1)^{i} d_{A}\right)$ be the translated complex. Then this becomes $a \mathbb{C}$-Hodge complex when endowed with filtrations $F, \widehat{F}$ and $W^{\bullet}[i]=W^{\bullet+i}$. For any pair of integers the twist $A(a, b)$ which is $A$ with the filtrations $F^{\bullet}[a], \widehat{F}^{\bullet}[b]$ and $W^{\bullet}[-a-b]$ is a $\mathbb{C}$-Hodge complex. $A(1,1)$ is the Tate twist.

Example 2.3. Let $X$ be a compact Kähler manifold. The de Rham complex $\mathcal{E}^{\bullet}(X)$ with filtrations

$$
\begin{gathered}
F^{p} \mathcal{E}^{\bullet}(X)=\bigoplus_{p^{\prime} \geq p} \mathcal{E}^{p^{\prime} q}(X) \\
\widehat{F}^{q} \mathcal{E}^{\bullet}(X)=\bigoplus_{q^{\prime} \geq q} \mathcal{E}^{p q^{\prime}}(X) \\
W_{0} \mathcal{E}^{\bullet}(X)=\mathcal{E}^{\bullet}(X) ; W_{-1} \mathcal{E}^{\bullet}(X)=0
\end{gathered}
$$

is a $\mathbb{C}$-Hodge complex by classical Hodge theory.

Example 2.4. Let $X$ be as above. A polarized complex variation of Hodge structure is a $C^{\infty}$ vector bundle $V$ with an indefinite $C^{\infty}$ Hermitian metric $<,>, a$ decomposition $V=\bigoplus_{p+q=w} V^{p q}$, and a flat connection $\nabla$ on $V$ subject to the following axioms:

- The metric is positive definite on $V^{p q}$ for $p$ even and negative definite when $p$ is odd, and the decomposition is orthogonal.

- The connection is compatible with the metric (i.e. $\nabla$ satisfies the Leibniz rule with respect to $<,>$ ) and satisfies the following form of Griffith's transversality:

$$
\nabla\left(V^{p q}\right) \subseteq \mathcal{E}^{01}\left(V^{p+1, q-1}\right) \oplus \mathcal{E}^{10}\left(V^{p, q}\right) \oplus \mathcal{E}^{01}\left(V^{p, q}\right) \oplus \mathcal{E}^{10}\left(V^{p-1, q+1}\right)
$$

where $\mathcal{E}^{r s}(U)$ is the sheaf $C^{\infty} U$-valued rs-forms. 
The de Rham complex $\left(\mathcal{E}^{\bullet}(X, V), \nabla\right)$ becomes a $\mathbb{C}$-Hodge complex when equipped with the filtrations

$$
\begin{gathered}
F^{p} \mathcal{E}^{\bullet}(X, V)=\bigoplus_{p^{\prime}+p^{\prime \prime} \geq p} \mathcal{E}^{p^{\prime} q^{\prime}}\left(X, V^{p^{\prime \prime} q^{\prime \prime}}\right) \\
\widehat{F}^{q} \mathcal{E}^{\bullet}(X)=\bigoplus_{q^{\prime}+q^{\prime \prime} \geq q} \mathcal{E}^{p^{\prime} q^{\prime}}\left(X, V^{p^{\prime \prime} q^{\prime \prime}}\right) \\
W_{w} \mathcal{E}^{\bullet}(X)=\mathcal{E}^{\bullet}(X, V) ; W_{w-1} \mathcal{E}^{\bullet}(X)=0 .
\end{gathered}
$$

This follows from the generalized Kähler identities of Deligne and Simpson [S], Z. A lattice is a locally constant sheaf $\mathcal{V}$ of Abelian groups with finitely generated stalks and an isomorphism $\mathcal{V} \otimes \mathbb{C} \cong k e r(\nabla)$ such that $V^{p q}$ is conjugate to $V^{q p}$ with respect to resulting $\mathbb{R}$-structure. The usual notion of a polarized variation of Hodge structure is equivalent to a complex variation of Hodge structure and a lattice. See D3] [GS], [S] for more information.

Complex (usually nonintegral) variations of Hodge structure, occur naturally in Simpson's work [S] as fixed points in the moduli space of Higgs bundles under the natural $\mathbb{C}^{*}$-action. Using this, he has shown that any representation of the fundamental group of a smooth projective variety can be deformed to the monodromy of a complex variation of Hodge structure.

In the previous examples, the complexes arose by taking global sections of complexes of sheaves. This can be generalized as follows. A $\mathbb{C}$-Hodge complex on a space $X$ is a quadruple $\left(A^{\bullet}, F^{\bullet}, \widehat{F}^{\bullet}, W^{\bullet}\right)$ consisting of a bounded below complex of sheaves of $\mathbb{C}$-vector spaces $A^{\bullet}$ with finite dimensional cohomology, together with three biregular filtrations on it such that:

$\mathbb{H C} 1$. $A^{\bullet}$ has finite dimensional hypercohomology.

$\mathbb{H} \mathbb{C} 2$. For each $p$, the filtrations on $\mathbb{R} \Gamma G r_{W}^{p} A$ induced by $F$ and $\widehat{F}$ are strictly preserved by the differentials.

$\mathbb{H} \mathbb{C}$ 3. For every $i, p, F$ and $\widehat{F}$ induce a pure $\mathbb{C}$-Hodge structure of weight $i-p$ on $\mathbb{H}^{i}\left(G r_{W}^{p} A\right)$.

A map of trifiltered complexes

$$
\left(A^{\bullet}, F^{\bullet} A^{\bullet}, \widehat{F}^{\bullet} A^{\bullet}, W^{\bullet} A^{\bullet}\right) \rightarrow\left(B^{\bullet}, F^{\bullet} B^{\bullet}, \widehat{F}^{\bullet} B^{\bullet}, W^{\bullet} B^{\bullet}\right)
$$

will be called an acyclic resolution if the associated maps

$$
G r_{F A}^{\bullet} G r_{W A}^{\bullet} A^{\bullet} \rightarrow G r_{F B}^{\bullet} G r_{W B}^{\bullet} B^{\bullet}
$$

and

$$
G r_{\hat{F} A}^{\bullet} G r_{W A}^{\bullet} A^{\bullet} \rightarrow G r_{\hat{F} B}^{\bullet} G r_{W B}^{\bullet} B^{\bullet}
$$

are acyclic resolutions in the usual sense. Canonical acyclic resolutions can be constructed using Godement's flasque resolutions. The axioms imply that if $\left(A^{\bullet}, F^{\bullet}, \widehat{F}^{\bullet}, W^{\bullet}\right)$ is a $\mathbb{C}$-Hodge complex, then after replacing it by an acyclic resolution, $\Gamma\left(A^{\bullet}\right)$ with its induced filtrations is a $\mathbb{C}$-Hodge complex in the earlier sense.

Example 2.5. Let $D \subset X$ be a divisor with normal crossings in a compact Kähler manifold and let $j: X-D \hookrightarrow X$ be the inclusion. Let $A_{X}^{\bullet}$ be the sheaf of $\mathbb{C}$ valued real analytic differential forms on $X$. Following Navarro Aznar $[\mathbb{N}]$, define $A_{X}^{\bullet}(\log D)$ to be the $A_{X}^{0}$-subalgebra of $j_{*} A_{X-D}^{\bullet}$ generated by $A_{X}^{\bullet}$ and $\log |f|$, Redf/f, and Im $d f / f$ for any (local) holomorphic function $f$ vanishing along a 
component of $D$. This is a subcomplex of $j_{*} A_{X-D}^{\bullet}$, which is an incarnation of $\mathbb{R} j_{*} \mathbb{C}$. Furthermore it has a bigrading

$$
A_{X}^{n}(\log D)=\bigoplus_{p+q=n} A_{X}^{p, q}(\log D)
$$

by $(p, q)$ type. With filtrations

$$
\begin{gathered}
F^{p} A_{X}^{\bullet}(\log D)=\bigoplus_{p^{\prime} \geq p} A_{X}^{p^{\prime}, q}(\log D) \\
\widehat{F}^{q} A_{X}^{\bullet}(\log D)=\bigoplus_{q^{\prime} \geq q} A_{X}^{p, q^{\prime}}(\log D) \\
W_{k} A_{X}^{m}(\log D)=\operatorname{image}\left(A_{X}^{k}(\log D) \wedge A_{X}^{m-k} \rightarrow A_{X}^{m}(\log D)\right)
\end{gathered}
$$

$A_{X}(\log D)$ becomes a $\mathbb{C}$-Hodge complex. The complex $A_{X}^{\bullet}(\log D)$ can be replaced by the $C^{\infty}$ analogue constructed by Burgos $[\mathrm{Bu}]$ to obtain an explicit acyclic resolution of this $\mathbb{C}$-Hodge complex.

Example 2.6. Let $X$ and $D$ be as above. Let $\left(V^{o}, \nabla^{o}\right)$ be a holomorphic bundle on $X-D$ with a flat connection which is unitary in the sense that $\mathcal{V}=k \operatorname{er}\left(\nabla^{o}\right)$ corresponds to a unitary representation of $\pi_{1}(X-D)$. Then there exists a unique extension of $V^{o}$ to a vector bundle $V$ on $X$ such that $\nabla^{o}$ extends to a logarithmic connection $\nabla$ with residues having eigenvalues in $[0,1)$ [D1]. The complex $\left(A_{X}^{\bullet}(\log D) \otimes V, \nabla\right)$ is quasi-isomorphic to $\mathbb{R} j_{*} \mathcal{V}$. There is a filtration $W$ on $\left(A_{X}^{\bullet}(\log D) \otimes V, \nabla\right)$, such that together with the filtrations

$$
\begin{aligned}
& F^{p} A_{X}^{\bullet}(\log D) \otimes V=\bigoplus_{p^{\prime} \geq p} A_{X}^{p^{\prime}, q}(\log D) \otimes V \\
& \widehat{F}^{q} A_{X}^{\bullet}(\log D) \otimes V=\bigoplus_{q^{\prime} \geq q} A_{X}^{p, q^{\prime}}(\log D) \otimes V
\end{aligned}
$$

this becomes a $\mathbb{C}$-Hodge complex [A, IV.1].

Example 2.7. A morphism of $\mathbb{C}$-Hodge complexes is a map of complexes which is compatible with the filtrations. Let $f:\left(A^{\bullet}, F^{\bullet} A^{\bullet}, \ldots\right) \rightarrow\left(B^{\bullet}, F^{\bullet} B^{\bullet}, \ldots\right)$ be a morphism. The mapping cone is a new complex Cone $(f)^{\bullet}=C^{\bullet}$ with

$$
C^{n}=A^{n+1} \oplus B^{n}
$$

and $d(\alpha, \beta)=(-d \alpha, d \beta-f(\alpha))$. With the filtrations

$$
\begin{aligned}
& F^{p} C^{n}=F^{p} A^{n+1} \oplus F^{p} B^{n} \\
& \widehat{F}^{p} C^{n}=\widehat{F}^{p} A^{n+1} \oplus \widehat{F}^{p} B^{n}
\end{aligned}
$$

and

$$
W_{k} C^{n}=W_{k-1} A^{n+1} \oplus W_{k} B^{n}
$$

$C \bullet$ becomes a $\mathbb{C}$-Hodge complex. By construction there are morphisms $B \rightarrow$ Cone $(f)$ and Cone $(f) \rightarrow A[1]$ where $A[1]$ is defined in example 2.2

The previous example can be generalized. 
Example 2.8. A differential graded $\mathbb{C}$-Hodge complex consists of a collection $\left(A^{n \bullet}, F A^{n \bullet}, \ldots\right)$ of $\mathbb{C}$-Hodge complexes, and morphisms $\delta_{n}: A^{n, \bullet} \rightarrow A^{n+1, \bullet}$ satisfying $\delta_{n+1} \delta_{n}=0$. Define the total (or associated single) complex $T^{\bullet}=$ tot $^{\bullet}(A)$ by

$$
T^{n}=\bigoplus_{i+j=n} A^{i j}
$$

with differential $d+(-1)^{j} \delta_{j}$. With filtrations

$$
\begin{aligned}
& F^{p} T^{n}=\bigoplus_{i+j=n} F^{p} A^{i j} \\
& \widehat{F}^{p} T^{n}=\bigoplus_{i+j=n} \widehat{F}^{p} A^{i j}
\end{aligned}
$$

and

$$
W_{k} T^{n}=\bigoplus_{i+j=n} W_{k+i} A^{i j}
$$

$T^{\bullet}$ becomes a $\mathbb{C}$-Hodge complex.

The above constructions will be used extensively later. Here are a few concrete instances.

Example 2.9. Let $X$ be a compact Kähler manifold, $i: Y \hookrightarrow X$ a closed submanifold and $j: U=X-Y \hookrightarrow X$ the the complement. Let $C^{\bullet}$ be the mapping cone of the restriction map $\mathcal{E}_{X}^{\bullet} \rightarrow \mathcal{E}_{Y}^{\bullet}$. This is a $\mathbb{C}$-Hodge complex quasi-isomorphic to $j_{!} \mathbb{C}_{U}$, and $H^{i}\left(j ! \mathbb{C}_{U}\right) \cong H^{i}(X, Y)$.

The above example is very special case of the constructions of the final section.

Example 2.10. Let $X$ be a compact Kähler manifold, and let $\theta \in H^{0}\left(X, \Omega_{X}^{1}\right)$ and $N$ be some positive integer. Let $\theta^{\prime}$ denote the operator $\phi \mapsto(-1)^{\operatorname{deg} \phi} \theta \wedge \phi$. Then $\left(\theta^{\prime}\right)^{2}=0$, we get a differential graded $\mathbb{C}$-Hodge complex

$$
\mathcal{E}_{X}^{\bullet} \stackrel{\theta^{\prime}}{\longrightarrow} \mathcal{E}_{X}^{\bullet}(1,0)[1] \stackrel{\theta^{\prime}}{\longrightarrow} \ldots \mathcal{E}_{X}^{\bullet}(N, 0)[N]
$$

where $\mathcal{E}_{X}(i, 0)[i]$ is defined in 2.9 and 2.9.

This example is from [A]. Related constructions occur in the work of Steenbrink St.

In example 2.1, the cohomology groups of $A^{\bullet}$ carry $\mathbb{C}$-Hodge structures with filtrations induced by $F, \widehat{F}$ and $W$, because the category of $\mathbb{C}$-Hodge structures is Abelian. This generalizes to all $\mathbb{C}$-Hodge complexes. The following, which was announced in [A] and is an analogue of [D2, scholium 8.1.9], is the basic result.

Theorem 2.11. Let $\left(A^{\bullet}, F^{\bullet}, \widehat{F}^{\bullet}, W^{\bullet}\right)$ be a $\mathbb{C}$-Hodge complex then

(a) The spectral sequence induced by $W$ degenerates at $E_{2}$.

(b) $H^{i}\left(A^{\bullet}\right)$ carries a $\mathbb{C}$-Hodge structure with filtrations given by $F^{\bullet}, \widehat{F}^{\bullet}$ and $W^{\bullet}$ shifted by $i$, or explicitly by:

$$
\begin{gathered}
F^{p} H^{i}\left(A^{\bullet}\right)=\operatorname{image}\left[H^{i}\left(F^{p} A^{\bullet}\right) \rightarrow H^{i}\left(A^{\bullet}\right)\right] \\
\widehat{F}^{p} H^{i}\left(A^{\bullet}\right)=\operatorname{image}\left[H^{i}\left(\widehat{F}^{p} A^{\bullet}\right) \rightarrow H^{i}\left(A^{\bullet}\right)\right] \\
W^{k} H^{i}\left(A^{\bullet}\right)=\text { image }\left[H^{i}\left(W^{k+i} A^{\bullet}\right) \rightarrow H^{i}\left(A^{\bullet}\right)\right]
\end{gathered}
$$

(c) The spectral sequences associated to $F$ and $\widehat{F}$ degenerate at $E_{1}$. 
The proof of the above theorem will be given in Section 3. A variant of part (a), proved by a similar argument, is:

Proposition 2.12. The spectral sequence induced by $W^{\bullet}$ on $G r_{F}^{i} A^{\bullet}$ degenerates at $E_{2}$.

Analogues of (c) also hold, for $F$ and $\widehat{F}$, namely the spectral sequences induced by $F^{\bullet}$ and $\widehat{F}^{\bullet}$ on $G r_{W}^{i} A^{\bullet}$ degenerate at $E_{1}$. This is nothing but a restatement of axiom $\mathrm{HC} 1$. As a corollary, one gets similar statements for $\mathbb{C}$-Hodge complexes on topological space with cohomology replaced by hypercohomology.

The degeneration statements have a number of important applications. When part (c) of the theorem is applied to example 2.6, along with the filtered quasiisomorphism $\left(\Omega_{X}^{\bullet}(\log D) \otimes V, F^{\bullet}\right) \hookrightarrow\left(A_{X}^{\bullet}(\log D) \otimes V, F^{\bullet}\right)$, one obtains the degeneration of the spectral sequence

$$
H^{q}\left(\Omega_{X}^{p}(\log D) \otimes V\right) \Rightarrow H^{p+q}(X-D, \mathcal{V})
$$

This fact, and its generalizations, can be used to prove vanishing theorems (see EV). For example, if $X-D$ is Stein (e.g. if $X$ is projective and $D$ ample) then it has the homotopy type of a CW-complex of dimension at most $n=\operatorname{dim} X$; this implies the following version of Kodaira's vanishing theorem:

$$
H^{i}\left(X, \omega_{X}(D) \otimes V\right) \subseteq H^{n+i}(X-D, \mathcal{V})=0
$$

for $i>0$. When proposition 2.12 is applied to the complex of example 2.10, one recovers a theorem of Green and Lazarsfeld GL that the spectral sequence

$$
E_{1}=H^{q}\left(X, \Omega_{X}^{p}\right) \Rightarrow \mathbb{H}^{p+q}\left(X ; O_{X} \stackrel{\theta}{\longrightarrow} \Omega_{X}^{1} \stackrel{\theta}{\longrightarrow} \ldots\right)
$$

degenerates at $E_{2}$. This kind of result, while technical, is crucial for establishing their generic vanishing theorems. The argument is adapted from $\mathrm{A}$ which contains further details and refinements.

With the basic homological apparatus in place, the integral structure can now be added. An integral structure for a $\mathbb{C}$-Hodge complex $\left(A^{\bullet}, W, F, \widehat{F}\right)$ on a topological space consists of a bounded below complex of sheaves of Abelian groups $A_{\mathbb{Z}}^{\bullet}$ with finitely generated cohomology and quasi-isomorphisms

$$
A_{\mathbb{Z}}^{\bullet} \otimes \mathbb{Q} \stackrel{\sim}{\leftarrow} A^{\prime \bullet} \stackrel{\sim}{\rightarrow} A_{\mathbb{C}}^{\bullet}
$$

inducing lattices on cohomology. Note that the intermediate complex $A^{\prime \bullet}$ is part of the data (or to put it another way: fix an explicit representative for an isomorphism $A_{\mathbb{Z}}^{\bullet} \otimes \mathbb{Q} \cong A_{\mathbb{Q}}^{\bullet}$ in the derived category ). The easist way to ensure that $W_{\bullet} H^{\bullet}\left(A^{\bullet}\right)$ is rational is to construct a filtered complex of $\mathbb{Q}$-vector spaces $\left(A_{\mathbb{Q}}^{\bullet}, W_{\mathbb{Q}}\right)$ and isomorphisms

$$
\begin{gathered}
A_{\mathbb{Z}}^{\bullet} \otimes \mathbb{Q} \cong A_{\mathscr{Q}}^{\bullet} \\
\left(A_{\mathbb{Q}}^{\bullet}, W_{\mathbb{Q}}\right) \otimes \mathbb{C} \cong\left(A^{\bullet}, W\right)
\end{gathered}
$$

in the derived and filtered derived categories. A Hodge complex will consist of a $\mathbb{C}$ Hodge complex together with a choice of integral structure. Hodge complexes carry mixed Hodge structures on cohomology. A trivial example of an integral structure is the inclusion of the constant sheaf $\mathbb{Z}_{X} \subset \mathcal{E}_{X}^{\bullet}$ into the De Rham complex of a compact Kähler manifold. More generally for variations of Hodge structure, the inclusion of the lattice in $\mathcal{E}_{X}^{\bullet}(V)$ supplies an integral structure. In example 2.5, $A_{\mathbb{Z}}$ can be taken to be the direct image under $j_{*}$ of the complex of sheaves of singular $C^{\infty}$ cochains, $A^{\prime}=A=A_{X}^{\bullet}(\log D)$, and $A^{\prime} \rightarrow A_{\mathbb{Z}} \otimes \mathbb{C}$ the map which assigns to a 
form $\alpha$, the cochain $\gamma \mapsto \int_{\gamma} \alpha$ (the rationality of $W$ is a consequence of a filtered quasi-isomorphism $\left(A_{\mathbb{Z}}, \tau_{\leq}\right) \otimes \mathbb{C} \cong\left(A, W_{\bullet}\right)$, D2, 团).

A few remarks ought to be made about the relationship between Hodge complexes and (cohomological) mixed Hodge complexes [D2, 8.1]. The set up for the latter is similar, one has a collection of complexes with filtrations and compatability isomorphisms subject to appropriate conditions. Although I do not expect a precise equivalence, it does seem to be case in practice that whenever one has a natural Hodge complex, one has a corresponding cohomological mixed Hodge and conversely. However, there are interesting examples of $\mathbb{C}$-Hodge complexes (or structures) which do not possess any integral or even real structure. The $\mathbb{C}$ Hodge complexes of examples 2.4, 2.6 and 2.10 will not generally possess natural integral structures. For certain applications (such as those discussed above), the degeneration of the above spectral sequences is precisely what is required; the integral structure is irrelevant even when it exists. For these reasons, the notion of a $\mathbb{C}$-Hodge complex is useful on its own.

For any space $X$, the collection of $\mathbb{C}$-Hodge complexes forms a category, denoted $C_{\mathbb{C}-\text { Hodge }}(X)$. More generally, let $C_{\mathbb{C}-\text { Hodge }}$ be the category whose objects are pairs consisting of a topological space and a $\mathbb{C}$-Hodge complex on it. A morphism from $(X,(A, \ldots))$ to $(Y,(B, \ldots))$ is pair consisting of a continuous map $f: X \rightarrow Y$ and a map of trifiltered complexes $\left(f^{*} B, \ldots\right) \rightarrow(A, \ldots)$. This category is fibered over the category of topological spaces by the functor $|\ldots|$, and $C_{\mathbb{C}-H o d g e}(X)$ is the fiber over $X$. The category of Hodge complexes $C_{H o d g e}$ is defined similarly.

\section{Proof of Theorem 2.11}

Let $A^{\bullet}$ be a bounded below complex of vector spaces with two biregular filtrations $W^{\bullet}, F^{\bullet}$. There is a spectral sequence corresponding to the first filtration with

$$
E_{1}^{p q}(A, W)=H^{p+q}\left(G r_{W}^{p} A^{\bullet}\right)
$$

and differential

$$
d_{1}: E_{1}^{p q}(A, W) \rightarrow E_{1}^{p+1, q}(A, W)
$$

given by the connecting map associated to

$$
0 \rightarrow G r_{W}^{p+1} A \rightarrow W^{p} A / W^{p+2} A \rightarrow G r_{W}^{p} A \rightarrow 0 .
$$

This can be filtered by $F$ in many (usually) different ways. The first direct filtration is

$$
F_{\text {dir }}^{i} E_{r}^{p q}(A, W)=\text { image }\left[E_{r}^{p q}\left(F^{i}, F^{i} \cap W\right) \rightarrow E_{r}^{p q}(A, W)\right] .
$$

The recursive filtration is defined inductively by $F_{r e c}^{i} E_{1}^{p q}=F_{d i r}^{i} E_{1}^{p q}$ and

$$
F_{r e c}^{i} E_{r+1}^{p q}(A, W)=\operatorname{image}\left[F_{r e c}^{i} \cap k e r_{r}^{p q}(A, W)\right]
$$

where

$$
\operatorname{ker}_{r}^{p q}(A, W)=\operatorname{ker}\left[E_{r}^{p q}(A, W) \rightarrow E_{r}^{p+r, q-r+1}(A, W)\right]
$$

and the image is taken in $E_{r+1}^{p q}(A, W) . E_{\infty}$ is a subquotient of $H^{*}(A)$ and it inherits a filtration induced by

$$
F^{i} H^{*}(A)=\operatorname{image}\left[H^{*}\left(F^{i} A\right) \rightarrow H^{*}(A)\right]
$$

which will be called the final filtration. The first direct filtration is useful because it is preserved by differentials $d_{r}: E_{r}^{p q}(A, W) \rightarrow E_{r}^{p+r, q-r+1}(A, W)$, while the recursive filtration is what one is seeing if one follows the spectral sequence from $E_{1}$ 
to $E_{2}$ to ... Fortunately in situations arising Hodge theory, agreement is guaranteed by:

Theorem 3.1. (D2, 1.3.16,1.3.17, 7.2.5]). If $d_{i}$ strictly preserve $F_{\text {dir }}$ for $i=$ $0,1 \ldots r$, then the first direct and recursive filtrations on $E_{r+1}$ coincide. If $r=\infty$ then these filtrations coincide with the final filtration on $E_{\infty}$.

Remark 3.2. The above condition for $i=0$ just says that the filtration on $G r_{W}^{\bullet} A$ induced by $F$ is strict.

Proof of theorem 2.11. The argument goes along the lines presented in D2. Axiom HC3 and the exactness of $G r_{F}$ and $G r_{\hat{F}}$ on the category of $\mathbb{C}$-Hodge structures implies that $d_{1}$ strictly preserves $F_{d i r}$ and $\widehat{F}_{d i r}$. The same goes for $d_{0}$ by HC2. Thus the first direct and recursive filtrations for $F$ and $\widehat{F}$ on $E_{2}(A, W)$ coincide. Therefore $E_{2}^{p q}(A, W)$, when equipped with these filtrations, becomes a pure $\mathbb{C}$ Hodge structure of weight $q$ because it is a subquotient of $E_{1}^{p q}(A, W)$. Consequently $d_{2}: E_{2}^{p q}(A, W) \rightarrow E_{2}^{p+2, q-1}(A, W)$ is a morphism of Hodge structures of different weights and so it must vanish. As zero strictly preserves everything, the first direct and recursive filtrations on $E_{3}$ agree, then by similar reasoning $d_{3}=0$. Continuing in this fashion proves (a).

It follows from the previous paragraph that $F$ and $\widehat{F}$ induce weight $i-k \mathbb{C}$-Hodge structures on $G r_{W}^{k} H^{i}\left(A^{\bullet}\right)$. This implies (b).

The proof of the last part is based on the fact that given a spectral sequence of finite dimensional vector spaces (lying in a translate of the first quadrant), there is an inequality

$$
\sum_{p+q=n} \operatorname{dim} E_{r}^{p q} \geq \operatorname{dim} H^{n}\left(A^{\bullet}\right)
$$

and equality holds if and only if $E_{r}^{p q}=E_{\infty}^{p q}$ for all $p, q$. It suffices to prove the opposite inequality for $E_{r}=E_{1}(A, F)$. Applying the above inequality for a different spectral sequence gives

$$
\sum_{q} \operatorname{dim} E_{2}^{q, n-q}\left(G r_{F}^{p} A, W\right) \geq \operatorname{dim} H^{n}\left(G r_{F}^{p} A\right)=\operatorname{dim} E_{1}^{p, n-p}(A, F) .
$$

There is a commutative diagram

$$
\begin{array}{cccc}
\cdots & E_{1}^{q, n-q}\left(G r_{F}^{p} A, W\right) & \rightarrow & E_{1}^{q+1, n-q}\left(G r_{F}^{p} A, W\right) \\
\cdots & H^{n}\left(G r_{F}^{p} G r_{W}^{q}\right) & \rightarrow & H^{n+1}\left(G r_{F}^{p} G r_{W}^{q+1}\right) \\
& \cdots & & \| \\
\cdots & G r_{F}^{p} E_{1}^{q, n-q}(A, W) & \rightarrow & G r_{F}^{p} E_{1}^{q+1, n-q}(A, W)
\end{array}
$$

where the top isomorphisms follows from Zassenhaus' lemma [D2, 1.2.1], and the bottom from axiom HC1. As $E_{1}(A, W)$ carries a $\mathbb{C}$-Hodge structure and $G r_{F}$ is exact for these, it follows that

$$
E_{2}^{q, n-q}\left(G r_{F}^{p} A, W\right) \cong G r_{F}^{p} E_{2}^{q, n-q}(A, W) .
$$

By (a),

$$
\sum_{q} \operatorname{dim} E_{2}^{q, n-q}(A, W)=\operatorname{dim} H^{n}\left(A^{\bullet}\right)
$$


and hence

$$
\sum_{p} \operatorname{dim} E_{1}^{p, n-p}(A, F) \leq \sum_{p, q} \operatorname{dim} G r_{F}^{p} E_{2}^{q, n-q}(A, W)=\operatorname{dim} H^{n}\left(A^{\bullet}\right)
$$

as required. The argument for $\widehat{F}$ is identical.

\section{Functorial Hodge Structures}

Now I will address the basic problem of constructing functorial mixed Hodge structures on the cohomology of a given category of spaces and sheaves. The obvious strategy is to produce a functorial Hodge complex for each pair under consideration, but this is usually very hard to implement. A more useful approach is to construct explicit complexes for special pairs, and then try and approximate the general pairs by the special ones.

Here is the precise set up. Suppose that $V$ is a category with pairwise fibered products, a terminal object, and a "forgetful" functor (denoted by $|\ldots|$ ) to the category Top of topological spaces which preserves these operations. Assume furthermore that for each $X \in O b(V)$ there is a category $\mathcal{C F}(X)$ equipped with a forgetful functor (also denoted by $|\ldots|$ ) to the category $C^{+}(|X|)$ of bounded below complexes of sheaves of Abelian groups on $|X|$. For each morphism $f: X \rightarrow Y$, there is a pullback functor $f^{*}: \mathcal{C F}(Y) \rightarrow \mathcal{C F}(X)$ compatible with $f^{*}$ for sheaves. One should think of an element of $\mathcal{C F}(X)$ as a complex of sheaves on $X$ enriched with additional data. $|X|$ will be denoted by $X$ if there is no danger of confusion. Let $\mathcal{C F}$ be the category of pairs $X \in O b(V), \mathcal{F} \in \mathcal{C F}(X)$, a morphism of pairs is a pair of morphisms $\left(f: X_{1} \rightarrow X_{2}, f^{*} \mathcal{F}_{2} \rightarrow \mathcal{F}_{1}\right)$. There is an obvious functor from $\mathcal{C F} \rightarrow V$ and $\mathcal{C F}(X)$ is just the fiber over $X \in O b(V) . \mathcal{C F}$ will be called a family of coefficients. I will say that $\mathcal{C F}$ is an Abelian family of coefficients if each $\mathcal{C F}(X)$ is an Abelian category and the functors $f^{*}$ and $|\ldots|$ are exact.

Define a Hodge complex on the family of coefficients $\mathcal{C F}$ to be a functor $A$ : $\mathcal{C F} \rightarrow C_{\text {Hodge }}$ such that

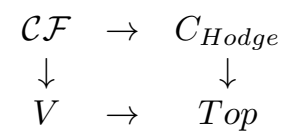

commutes, and a natural quasi-isomorphism between the composite of $A$ with $C_{\text {Hodge }} \rightarrow C^{+}(-)$and $|\ldots|$. In other words, it is just a functorial choice of a Hodge complex on $|X|$ naturally quasi-ismorphic to $\mathcal{F}$, for each pair $(X, \mathcal{F})$ with $X \in V$ and $\mathcal{F} \in \mathcal{C F}(X)$. The definition of a $\mathbb{C}$-Hodge complex on $\mathcal{C F}$ is similar.

Lemma 4.1. Given a $\left(\mathbb{C}\right.$-)Hodge complex on $\mathcal{C F}, H^{i}(|X|,|\mathcal{F}|)$ carries a $(\mathbb{C}$-)mixed structure which is functorial in both variables.

This lemma is somewhat limited in applicability, because it's hard to construct Hodge complexes on all of $\mathcal{C F}$. In order to formulate a more useful result, let me call a full subcategory $\Delta$ of $V$ dense provided that:

1. Given any object $Y \in O b V$, there is a morphism $X \rightarrow Y$ with $X \in \Delta$ such that the underlying map on spaces is proper and surjective. Call this a resolution of $Y$ in $\Delta$. 
2. Given a morphism $X \rightarrow Y$ and a proper surjective morphism $Y^{\prime} \rightarrow Y$, there is a resolution $X^{\prime} \rightarrow X$ which fits into a commutative diagram:

$$
\begin{array}{ccc}
X^{\prime} & \rightarrow & Y^{\prime} \\
\downarrow & & \downarrow \\
X & \rightarrow & Y
\end{array}
$$

(It's convenient to phrase the definition this way, even though the second condition implies the first. ) The notion of denseness is extended to coefficients in the most straightforward possible way. The preimage of $\Delta$ under $\mathcal{C F} \rightarrow V$ will be called a subfamily of coefficients; it is called dense if $\Delta$ is dense. The reason for introducing these notions comes from:

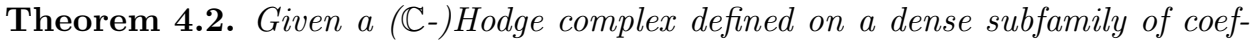
ficients, $H^{\bullet}(|X|,|\mathcal{F}|)$ carries a bifunctorial $(\mathbb{C}$-)mixed Hodge structure for all pairs $(X, \mathcal{F}) \in O b \mathcal{C F}$. If $\mathcal{C F}$ is an Abelian family of coefficients then any short exact sequence of coeffients induces a long exact sequence of mixed Hodge structures on cohomology.

An outline of the proof of 4.2 will be given in 1 .

Example 4.3. Let $V$ be the category of proper complex varieties. Let $\Delta$ be the full subcategory of nonsingular projective varieties. Density is deduced from either Hironaka's theorem [H]] (and Chow's lemma), or the easier theorem of de Jong [d] on the existence of nonsingular alterations. For $\mathcal{C F}(X)$, one can take the category consisting of the constant sheaf $\mathbb{Z}_{X}$ and the identity. A Hodge complex on $\Delta$ is given by 2.3. This yields the Deligne mixed Hodge structure on the cohomology of proper varieties (and indeed this is pretty much Deligne's original construction).

Example 4.4. Let $V$ and $\Delta$ be as above. Let $\mathcal{C F}(X)$ be the category pairs $(f$ : $X \rightarrow Y, \mathcal{F})$ where $f$ is a map to a smooth variety and $\mathcal{F}$ a polarized complex variation of Hodge structure on $Y$. Define $|(f, \mathcal{F})|=f^{*} \mathcal{F}$. The $\mathbb{C}$-Hodge complex of 2.4 yields $\mathbb{C}$-mixed Hodge structures on cohomologies of proper varieties with these coefficients.

The previous example is essentially due to Lasell [4] who used it in proving his generalization of Nori's Lefschetz theorem of $\pi_{1}$. One of the key steps amounts to verification that if $f: Z \rightarrow X$ is a morphism of smooth projective varieties (with $X$ and $Y=\operatorname{image}(f)$ connected) then

$$
\operatorname{ker}\left[H^{1}(X, \mathcal{V}) \rightarrow H^{1}(Y, \mathcal{V})\right]=\operatorname{ker}\left[H^{1}(X, \mathcal{V}) \rightarrow H^{1}\left(Z, f^{*} \mathcal{V}\right)\right]
$$

for any polarized complex variation of Hodge structure $\mathcal{V}$ on $X$. This follows rather formally from the existence and basic properties of the $\mathbb{C}$-Hodge structure on these cohomology groups.

Example 4.5. Let $V$ be the category of pairs $(X, Z)$ consisting of a proper complex variety $X$ and a Zariski closed subset $Z$. A morphism $\left(X_{1}, Z_{1}\right) \rightarrow\left(X_{2}, Z_{2}\right)$ is a morphism of the underlying schemes such that $Z_{1}$ is the preimage of $Z_{2} . \Delta$ is the full subcategory generated by pairs consisting of smooth projective varieties and divisors with normal crossings. Once again density follows from [Hi] or d. $\mathrm{dJ}$. The Hodge complex described in 2.5 (with the integral structure described in the discussion following theorem 2.11) yields the Deligne mixed Hodge structures on the cohomology of varieties with constant coefficients. 
Example 4.6. With the same $V$ and $\Delta$ as in the previous example, let $\mathcal{C F}(X, Z)$ be the category of unitary local systems on $X-Z$. This is an Abelian family of coefficients. Then the $\mathbb{C}$-Hodge complex of example 2.0 gives a $\mathbb{C}$-Hodge structure on the cohomology of varieties with coeffients in unitary local systems. This is compatible with the cohomology long exact sequence by the theorem, but it can be also deduced directly from the semisimplicity of $\mathcal{C F}(X, Z)$.

A more elaborate example will be given in section 6 .

\section{Cohomological Descent}

I will outline the proof of theorem 4.2. The name of the game is cohomological descent, which is a way of computing the cohomology of space in terms of the cohomology of a (simplicial) diagram of spaces lying over it. It is a generalization of Čech's procedure for computing cohomology, where the diagram in question is just the nerve of the open cover. Recall that a strict simplicial object in some category $C$ is a collection of objects $X_{n}$ indexed by the natural numbers, and "face" morphisms $\delta_{i}: X_{n} \rightarrow X_{n-1} i=0,1 \ldots n$ such that $\delta_{i} \delta_{j}=\delta_{j-1} \delta_{i}$ for $i<j$. I will usually drop the adjective "strict", although this is technically incorrect An augmentation to $X$ is a collection of morphisms $\epsilon_{n}: X_{n} \rightarrow X$ which commute with the face maps. A cosimplicial object is the dual notion where arrows go the other way, or equivalently a simplicial object in the opposite category. Suppose that $A^{0} \rightrightarrows A^{1} \ldots$ is a cosimplicial object in an additive category, then defining $d=\sum_{i}(-1)^{i} \delta_{i}$ results in a complex $A^{\bullet}$.

Suppose that $\epsilon_{\bullet}: X_{\bullet} \rightarrow X$ is an augmented simplicial space, i.e. an augmented simplicial object in the category of topological spaces. A sheaf on $X_{\bullet}$ is a collection of sheaves $F^{n}$ on $X_{n}$ and morphisms $\delta_{i}: \delta_{i}^{*} F^{n-1} \rightarrow F^{n}$ satisfying the simplicial identities. For example, given an augmentation $\epsilon_{\bullet}: X_{\bullet} \rightarrow X$ and a sheaf $F$ on $X$, the pullback $\epsilon_{\bullet}^{*} F$ with $\delta_{i}^{*}=i d$ is a sheaf on $X_{\bullet}$. Suppose that $F$ is a sheaf of Abelian groups. Then the Godement flasque resolution $\mathcal{G}^{\bullet}\left(F^{\bullet}\right)$ forms a complex of sheaves on $X_{\bullet}$. Taking direct images yields a cosimplicial object $\epsilon_{*}\left(\mathcal{G}^{\bullet}\left(F^{\bullet}\right)\right)$ in the category of complexes of sheaves on $X$. This in turn determines a double complex, and let $s \epsilon_{*}\left(\mathcal{G}^{\bullet}\left(F^{\bullet}\right)\right.$ ) denote the total or associated single complex (I am being a bit cavalier about signs; the important thing is that they be chosen in a consistent way so as to yielda complex). The map $\epsilon: X \bullet \rightarrow X$ satisfies cohomological descent if the natural map

$$
F \rightarrow s \epsilon_{*}\left(\mathcal{G}^{\bullet}\left(\epsilon^{*} F\right)\right)
$$

is quasi-isomorphism for any sheaf $F$ on $X$. In less prosaic terms, this says that the natural map $F \rightarrow \mathbb{R} \epsilon_{*} \epsilon^{*} F$ is an isomorphism in $D^{+}(X)$.

This phenomenon has already been encountered earlier in this article. Let

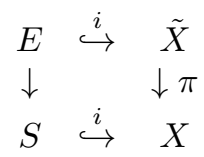

be the diagram given in proposition 1.1. Then the proof of that proposition actually showed:

\footnotetext{
${ }^{1}$ Strictly speaking, a simplicial object has additional degeneracy maps $X_{n-1} \rightarrow X_{n}$. In all our examples, $C$ will have finite coproducts, so we can turn a strict simplicial object $X \bullet$ in a true simplicial object $X_{n}^{+}$by inductively replacing $X_{n}$ by $X_{n} \amalg X_{n-1} \ldots$, one $X_{n-1}$ for each degeneracy map...
} 
Lemma 5.1.

$$
\underset{\operatorname{Ex}}{\rightarrow} \tilde{X} \coprod S \rightarrow X
$$

satisfies cohomological descent.

The next step is to find a general effective criterion for cohomological descent. Suppose that $X_{\bullet}$ is a simplicial set, and $x \in X_{n}$. If $x_{i}=\delta_{i} x$, then these satisfy $\delta_{j} x_{i}=\delta_{i} x_{j+1}$ for all $i \leq j$. Let me call such an $(n+1)$-tuple in $X_{n-1}$ an $n-1$ cycle, and let $Z_{n-1}\left(X_{\bullet}\right)$ denote the set of all of these. Note that $Z_{n-1}\left(X_{\bullet}\right)$ depends only on the maps $\delta_{i}: X_{n-1} \rightarrow X_{n-2}$. This observation will be useful for the inductive constructions later on. If $X \bullet \rightarrow X$ is an augmented simplicial set, then define $Z_{n-1}\left(X_{\bullet} \rightarrow X\right)$ by imposing the additional constraint that the $x_{i}$ take the same value in $X$. If $X_{\bullet} \rightarrow X$ is an augmented simplicial space then $Z_{n-1}\left(X_{\bullet} \rightarrow X\right)$ is also a topological space with a continuous map to $X$. Say that an augmented simplicial space $X_{\bullet} \rightarrow X$ is a hypercover if $X_{0} \rightarrow X$ and the obvious continuous maps $X_{n} \rightarrow Z_{n-1}\left(X_{\bullet} \rightarrow X\right)$ are proper and surjective for each $n$.

Theorem 5.2. A hypercover satisfies cohomological descent.

This result will be sufficient for our needs, although it is far from optimal: it does not include lemma 5.1 for example. For a more general statement and proofs, see [SGA4, V bis] or GNPP] (this last reference takes a slightly different point of view into which lemma 5.1 fits quite naturally). Note that these references use the stronger form of simplicial object having also degeneracy maps, but this will not affect the proofs since (in the notation of footnote 1) we can pass from $X \bullet \rightarrow X$ to $X_{\bullet}^{+} \rightarrow X$. Also, in order to convert our definition to those in the references, $Z_{n-1}\left(X_{\bullet}\right)$ is often written as $\left(\cos k s k_{n-1} X_{\bullet}\right)_{n}$.

Let $V$ be a category satisfying the conditions of the previous section and let $\Delta$ be a dense subcategory. Let Res be the set of resolutions in the sense of Section 4 i.e. morphisms with domain in $\Delta$ such that the underlying continuous map is proper and surjective. Given an augmented simplicial object $X \bullet \rightarrow X$ in the category $V$, $Z_{n-1}\left(X_{\bullet} \rightarrow X\right)$ can be defined as the object that represents the functor

$$
T \mapsto\left\{\left(f_{0}, \ldots f_{n}\right) \in \operatorname{Hom}\left(T, X_{n-1}\right)^{n+1} \mid \forall i \leq j, \delta_{j} f_{i}=\delta_{i} f_{j+1}\right\}
$$

in the category of objects over $X$ (the representability of this functor follows from our assumptions about $V)$. I will treat $Z_{n-1}\left(X_{\bullet} \rightarrow X\right)$ as an object of $V$ by forgetting the map to $X$. An augmented simplicial object $X_{\bullet} \rightarrow X$ in $V$, will be called a simplicial resolution of $X$ if $X_{0} \rightarrow X$ and each $X_{n} \rightarrow Z_{n-1}(X \bullet \rightarrow X)$ lies in Res. A simplicial resolution is a hypercover.

Theorem 5.3. Every object possesses a simplicial resolution. Given a hypercover $Y_{\bullet} \rightarrow Y$ and a morphism $X \rightarrow Y$, a simplicial resolution $X_{\bullet} \rightarrow X$ can be chosen so as to fit into a commutative diagram

$$
\begin{array}{ccc}
X \bullet & \rightarrow & Y \bullet \\
\downarrow & & \downarrow \\
X & \rightarrow & Y
\end{array}
$$

Any two simplicial resolutions are dominated by a third simplicial resolution.

\footnotetext{
${ }^{2}$ It would be more accurate to call this a hypercover for the Grothendieck topology of proper maps.
} 
In a nutshell, the proof is by induction. The key point is that once $X_{i}$ and the associated maps are defined for all $i<n$, then $Z_{n-1}\left(X_{\bullet} \rightarrow X\right)$ exists. And by its very construction, it carries morphisms to $X_{n-1}$ satisfying the appropriate face relations. Now take $X_{n}$ to be a resolution of $Z_{n-1}\left(X_{\bullet} \rightarrow X\right)$ (which fits into a commutative square

$$
\begin{array}{ccc}
X_{n} & \rightarrow & Y_{n} \\
\downarrow & & \downarrow \\
Z_{n-1}\left(X_{\bullet} \rightarrow X\right) & \rightarrow & Z_{n-1}\left(Y_{\bullet} \rightarrow Y\right)
\end{array}
$$

for the second part). To start the whole process, set $X_{0}$ equal to any resolution of $X$ (compatible with $Y_{0} \rightarrow Y$ ). For the last statement, apply the previous part with $Y=X$ and $Y_{\bullet}$ equal to the fiber product of two given simplicial resolutions of $X$.

Now, I can outline the proof of theorem 4.2. To simplify the discussion, let me dispense with the $\mathbb{Z}$-structure. Suppose that $(X, \mathcal{F}) \in O b \mathcal{C F}$, then choose a simplicial resolution $X_{\bullet} \rightarrow X$. The assumptions, guarantees that there are $\mathbb{C}$-Hodge complexes $\left(A^{\bullet n}(\mathcal{F}), W, F, \widehat{F}\right)$ quasi-isomorphic to $\epsilon_{n}^{*} \mathcal{F}$ on each $X_{n}$, and face morphisms $\delta_{i}$ between them. Replace these complexes by their Godement resolutions. This results in a differential graded $\mathbb{C}$-Hodge complex

$$
\Gamma\left(A^{0 \bullet}(\mathcal{F})\right) \stackrel{\delta_{0}-\delta_{1}}{\longrightarrow} \Gamma\left(A^{1 \bullet}(\mathcal{F})\right) \rightarrow \ldots
$$

The total complex $S^{\bullet}(\mathcal{F})$ is a $\mathbb{C}$-Hodge complex (example 2.8) which is naturally quasi-isomorphic to $\mathbb{R} \Gamma(\mathcal{F})$. This yields a $\mathbb{C}$-Hodge structure on $H^{\bullet}(X, \mathcal{F})$ which depends, apriori, on the choice of the simplicial resolution $X_{\bullet} \rightarrow X$. However given a second resolution $X_{\bullet}^{\prime} \rightarrow X$ dominating $X_{\bullet}$, one gets a morphism of the corresponding $\mathbb{C}$-Hodge structures which is an isomorphism of vector spaces and hence of Hodge structures, because the forgetful functor to vector spaces is exact and faithful. As any two simplicial resolutions are dominated by a third, this shows independence. Similarly since any morphism can be lifted to a morphism of simplicial resolutions, this proves functoriallity. Finally, suppose that $\mathcal{C F}$ is Abelian, and let

$$
0 \rightarrow \mathcal{F}_{1} \stackrel{f}{\rightarrow} \mathcal{F}_{2} \stackrel{g}{\rightarrow} \mathcal{F}_{3} \rightarrow 0
$$

be exact. Then there exists morphisms $S(f): S^{\bullet}\left(\mathcal{F}_{1}\right) \rightarrow S^{\bullet}\left(\mathcal{F}_{2}\right)$ and $S(g)$ : $S^{\bullet}\left(\mathcal{F}_{2}\right) \rightarrow S^{\bullet}\left(\mathcal{F}_{3}\right)$ of Hodge complexes. As $S(g) \circ S(f)=0$, there is a well defined map $\operatorname{Cone}\left(S^{\bullet}(f)\right)^{\bullet} \rightarrow S^{\bullet}\left(\mathcal{F}_{3}\right)$ which is a (nontrilftered) quasi-isomorphism. Therefore the $\mathbb{C}$-Hodge structures on cohomology coincide. The maps in the triangle

$$
S^{\bullet}\left(\mathcal{F}_{1}\right) \rightarrow S^{\bullet}\left(\mathcal{F}_{2}\right) \rightarrow \text { Cone }(f)^{\bullet} \rightarrow S^{\bullet}\left(\mathcal{F}_{1}\right)[1]
$$

yields maps of Hodge structures in the long exact sequence for cohomology.

The following construction is implicit in the above argument. Let $D_{\mathbb{C}-H o d g e}(p t)$ be obtained by localizing the category of $\mathbb{C}$-Hodge complexes $C_{\mathbb{C}-\text { Hodge }}(p t)$ with respect to (not necessarily filtered) quasi-isomorphisms (see [V] for the definition). Then this has the structure of a triangulated category with translation given in 2.2 and distinguished triangles represented by

$$
A^{\bullet} \stackrel{f}{\longrightarrow} B^{\bullet} \rightarrow \text { Cone }(f)^{\bullet} \rightarrow A^{\bullet}[1]
$$

where Cone $(f)$ is defined in 2.7. There is a $\Delta$-functor from $D_{\mathbb{C}-H o d g e}(p t)$ to the category of $\mathbb{C}$-Hodge structures which are compatible with the usual cohomology 
functor $H^{\bullet}: D^{+}(\mathbb{C}) \rightarrow \mathbb{C}-$ Mod. Similar remarks apply to the category of Hodge complexes. See [B] or [E] for further details about the construction.

\section{Constant Constructible Sheaves}

Recall that a sheaf of Abelian groups $\mathcal{F}$ on a complex variety $X$ is constructible if it has finitely generated stalks and there exists a partition of $X$ into Zariski locally closed subsets such that $\left.\mathcal{F}\right|_{U}$ is locally constant for each member $U$ of the partition. I will say that $\mathcal{F}$ is constant constructible if each restriction is in fact constant. As an illustration of the previous ideas, I want to prove?:

Theorem 6.1. Let $\mathcal{F}$ be a constant constructible sheaf on a proper complex variety $X$, then $H^{i}(X, \mathcal{F})$ carries a mixed Hodge structure which is functorial in both variables. A short exact sequence of sheaves induces a long exact sequence of mixed Hodge structures on cohomology.

Let $X$ be a variety over $\mathbb{C}$. A stratification is a finite Boolean algebra $\mathcal{B}$ of subsets of $X$ such that the Zariski closure of any element of $\mathcal{B}$ is also in $\mathcal{B}$. This somewhat unorthodox usage of terminology requires some explanation. Fix a stratification $\mathcal{B}$, and let $A(\mathcal{B})$ be the set of atoms or nonempty minimal elements. As $\mathcal{B}$ is a finite Boolean algebra, any nonempty element is a union of atoms. Let me define a partial order on $A$, by $Z_{1} \leq Z_{2}$ if and only if $\bar{Z}_{1} \subseteq \bar{Z}_{2}$. Then, one easily obtains

Lemma 6.2. If $Z \in A(\mathcal{B})$, then $\bar{Z}=\cup_{Z^{\prime} \leq Z} Z^{\prime}$ and $Z=\bar{Z}-\cup_{Z^{\prime}<Z} \bar{Z}^{\prime}$.

Consequently, $A(\mathcal{B})$ is a partition of $X$ into Zariski locally closed sets, and the elements of $\mathcal{B}$ are constructible. Conversely, given such a partition, the set of unions of elements of the partition yield a stratification.

Let $\mathcal{S} \mathcal{V}$ be the set of all pairs $(X, \mathcal{B})$ consisting of a variety with a stratification. This can be made into a category by taking as morphisms $f:\left(X_{1}, \mathcal{B}_{1}\right) \rightarrow\left(X_{2}, \mathcal{B}_{2}\right)$, morphisms $f: X_{1} \rightarrow X_{2}$ of varieties such that $f^{-1}(Z) \in \mathcal{B}_{1}$ for all $Z \in \mathcal{B}_{2}$. A morphism corresponding to the identity on the underlying variety is just a refinement of the stratification. A sheaf $\mathcal{F}$ on $X$ with finitely generated stalks will be called constant constructible with respect to a stratification $\mathcal{B}$ provided that $\left.\mathcal{F}\right|_{U}$ is constant for each $U \in A(\mathcal{B})$.

Given a (Weil) divisor $E$ on an irreducible variety $X$, let $\mathcal{B}(E)$ be the smallest stratification containing the irreducible components of $E$. If $X$ is smooth and $E$ a divisor with normal crossings, then $\mathcal{B}(E)$ will be refered to as a good stratification. Let $\Delta$ be the full subcategory of $\mathcal{S V}$ whose objects are disjoint unions of smooth projective varieties with good stratifications.

Proposition 6.3. SV satisfies the assumptions of section 4 , and $\Delta$ is dense.

Proof. We verify that $\mathcal{S} \mathcal{V}$ satisfies the axioms of $1 . \mathcal{S} \mathcal{V}$ certainly contains a terminal object. Given two morphisms $f_{i}:\left(X_{i}, \mathcal{B}_{i}\right) \rightarrow(Y, \mathcal{B})$ with $i=1$, 2 , let $P=\left(X_{1} \times_{Y}\right.$ $\left.X_{2}\right)_{\text {red }}$. Let $\mathcal{B}_{P}$ be the Boolean algebra generated by fibered products $\left(Z_{1} \times_{Y} Z_{2}\right)_{\text {red }}$ with $Z_{i} \in \mathcal{B}_{i}$. Then $\left(P, \mathcal{B}_{P}\right)$ is the fibered product of $f_{1}$ and $f_{2}$ in $\mathcal{S} \mathcal{V}$.

The next step is to show that every object possesses a resolution with respect to $\Delta$. If $(X, \mathcal{B})$ is a stratified variety, let $X^{\prime}$ be the disjoint union of the irreducible components. We can pull $\mathcal{B}$ back to $X^{\prime}$ to obtain a surjective proper morphism $\left(X^{\prime}, \mathcal{B}^{\prime}\right) \rightarrow(X, \mathcal{B})$. Since we can work with one component at a time, we may

\footnotetext{
${ }^{3}$ This argument was worked at MSRI during 1988-1989; my thanks for their support.
} 
assume that $X$ is irreducible. The proof will proceed by induction on the cardinality of $\mathcal{B}$. If $\mathcal{B}$ has less than or equal to 2 elements, in other words if the stratification is trivial, then the existence of a resolution follows immediately from [Hi] or dd]. In general, let $A$ be the set of atoms of $\mathcal{B}$. Choose an element $Z \in A$, which is minimal with respect to $\leq$. Then $Z$ is necessarily closed. Let $\mathcal{B}^{\prime} \subset \mathcal{B}$ be the Boolean subalgebra generated by the closures of elements of $A-\{Z\} . \mathcal{B}^{\prime}$ is easily seen to be a stratification not containing $Z$. Therefore, by induction, there is a resolution $\left(X_{1}, \mathcal{B}\left(E_{1}\right)\right) \rightarrow\left(X, \mathcal{B}^{\prime}\right)$. Let $Z_{1}$ be the preimage of $Z$. Then there exists a proper surjective morphism $X_{2} \rightarrow X_{1}$ from a second nonsingular variety such that the preimage $E_{2}$ of $Z_{1} \cup E_{1}$ is a divisor with normal crossings [loc. cit.]. Then $\left(X_{2}, \mathcal{B}\left(E_{2}\right)\right)$ is a resolution of $(X, \mathcal{B})$.

It remains to check the last condition for denseness. Given a morphism $\left(Y_{1}, \mathcal{B}_{Y_{1}}\right) \rightarrow$ $\left(Y_{2}, \mathcal{B}_{Y_{2}}\right)$ and a proper surjective morphism $\left(X_{2}, \mathcal{B}_{X_{2}}\right) \rightarrow\left(Y_{2}, \mathcal{B}_{Y_{2}}\right)$, let $\left(X_{1}, \mathcal{B}_{X_{1}}\right)$ be a resolution of the fibered product.

Given an object $(X, \mathcal{B})$ of $\mathcal{S} \mathcal{V}$, let $S(X, \mathcal{B})=\coprod_{U \in A(\mathcal{B})} U$ and let $J(X, \mathcal{B})$ : $S(X, \mathcal{B}) \rightarrow X$ denote the canonical map. Set

$$
T=T(X, \mathcal{B})=J(X, \mathcal{B})_{*} \circ J(X, \mathcal{B})^{*}: S h(X) \rightarrow S h(X),
$$

where $S h(X)$ is the category of sheaves on $X$. From the adjointness of $J_{*}$ and $J^{*}$, there are natural transformations:

$$
\eta: I \rightarrow T, \mu: T^{2} \rightarrow T
$$

satisfying the usual identities of a "triple" or monad [M]. Define the natural transformations:

$$
\delta_{i}=T^{i} \eta T^{n-i}: T^{n} \rightarrow T^{n+1}
$$

For any sheaf $\mathcal{F} \in S h(X)$, these maps determine a cosimplicial sheaf augmented over $\mathcal{F}$ :

$$
\mathcal{F} \rightarrow T \mathcal{F} \rightrightarrows T^{2} \mathcal{F} \ldots
$$

and hence also a complex (augmented over $\mathcal{F}$ ):

$$
\mathcal{F} \rightarrow T \mathcal{F} \stackrel{d}{\rightarrow} T^{2} \mathcal{F} \ldots
$$

where $d=\sum(-1)^{i} \delta_{i}$. Either of these will be denoted by $T^{\bullet} \mathcal{F}$ or $T(X, \mathcal{B})^{\bullet} \mathcal{F}$. This can be made more explicit as follows. Let $j_{U}: U \rightarrow X$ denote the inclusion for $U \in A(\mathcal{B})$. Since $j_{V}^{*} j_{U *}=0$ unless $V \leq U$, it follows that:

$$
T^{n} \mathcal{F}=\bigoplus_{U_{0} \leq \ldots \leq U_{n}} j_{U_{0} *}^{*} \ldots j_{U_{n} *}^{*} \mathcal{F}
$$

where I abbreviate $j_{U *} j_{U}^{*}$ by $j_{U *}^{*}$. The $i$ th face map $\delta_{i}$ is the sum of the adjunction maps:

$$
j_{U_{0} *}^{*} \ldots \hat{j}_{U_{i} *}^{*} \ldots j_{U_{n} *}^{*} \mathcal{F} \rightarrow j_{U_{0} *}^{*} \ldots j_{U_{n} *}^{*} \mathcal{F}
$$

Lemma 6.4. $T \cdot \mathcal{F}$ is quasi-isomorphic to $\mathcal{F}$.

Proof. Fix $x \in X$. It suffices to construct a functorial retraction $h:(T \mathcal{F})_{x} \rightarrow \mathcal{F}_{x}$ to the map $\mathcal{F}_{x} \rightarrow(T \mathcal{F})_{x}$, because then the augmented complex $\mathcal{F}_{x} \rightarrow\left(T^{\bullet} \mathcal{F}\right)_{x}$ carries a contracting homotopy defined by

$$
h:\left(T^{n+1} \mathcal{F}\right)_{x}=\left(T T^{n} \mathcal{F}\right)_{x} \rightarrow\left(T^{n} \mathcal{F}\right)_{x} .
$$


See [G, Appendix 5] for details. We have

$$
(T \mathcal{F})_{x}=\bigoplus_{U \in A(\mathcal{B})}\left(j_{U *}^{*} \mathcal{F}\right)_{x}
$$

If $x \in U$ then $\left(j_{U *}^{*} \mathcal{F}\right)_{x}=\mathcal{F}_{x}$, define $h$ to act as the identity on each summand $\left(j_{U *}^{*} \mathcal{F}\right)_{x}$ with $x \in U$, and zero on the other summands.

The above constructions are functorial. Given a morphism $f:(X, \mathcal{B}) \rightarrow\left(X^{\prime}, \mathcal{B}^{\prime}\right)$ and a sheaf $\mathcal{F}$ on $X^{\prime}$, there is a morphism of cosimplicial sheaves:

$$
f^{*} T\left(X^{\prime}, \mathcal{B}^{\prime}\right)^{\bullet} \mathcal{F} \rightarrow T(X, \mathcal{B})^{\bullet} f^{*} \mathcal{F} .
$$

Proof of theorem 6.1. Let $\mathcal{P S V}$ be the full subcategory of $\mathcal{S V}$ where the underlying variety is proper. The intersection $P \Delta=\Delta \cap O b \mathcal{P S V}$ is dense in $\mathcal{P S V}$. Let $\mathcal{C F}(X, \mathcal{B})$ denote the category of constant constructible sheaves on $(X, \mathcal{B}) \in$ $O b \mathcal{P S V}$. This determines an Abelian family of coefficients.

Let $(X, \mathcal{B})$ be an object of $P \Delta$, and let $\mathcal{F}$ be a constant constructible sheaf on it. There is no loss of generality in assuming that $X$ is connected, so that $\mathcal{B}$ is the Boolean algebra $\mathcal{B}(E)$ generated by the components of a divisor with normal crossings. In this case, the components of $T^{\bullet}(\mathcal{F})$ are fairly easy to describe: $j_{U_{0}}^{*} j_{U_{1} *}^{*} \ldots j_{U_{n} *}^{*} \mathcal{F}=j_{U_{0}}^{*} j_{U_{n} *}^{*} \mathcal{F}$ is a constant sheaf. Let

$$
A^{n \bullet}(\mathcal{F})=\bigoplus_{U_{0} \leq \ldots \leq U_{n}} j_{\bar{U}_{0} *}\left(\mathcal{E}_{\bar{U}_{0}}^{\bullet} \otimes j_{\bar{U}_{0}}^{*} j_{U_{n} *}^{*} \mathcal{F}, \ldots\right)
$$

be the associated Hodge complex. The differentials of $T^{\bullet}(\mathcal{F})$ extend to give a differential graded Hodge complex $A^{\bullet \bullet}(\mathcal{F})$. Then $\mathcal{F} \mapsto \operatorname{tot}^{\bullet}(A(\mathcal{F}))$ is a Hodge complex on $\left.\mathcal{C F}\right|_{P \Delta}$. The theorem now follows from theorem 4.2 .

There are a couple of variations on this construction. Call a sheaf $\mathcal{F}$ of $\mathbb{C}$-vector spaces unitary constructible if it has finite dimension stalks and there is stratification such that $\left.\mathcal{F}\right|_{U}$ is isomorphic to a unitary local system for each $U \in A(\mathcal{B})$. In this case, the sheaves $j_{U_{0}}^{*} j_{U_{1} *}^{*} \ldots j_{U_{n} *}^{*} \mathcal{F}$ are all unitary local systems, let $\left(V_{\left(U_{0}, \ldots U_{n}\right)}, \nabla\right)$ denote the extension of the associated flat vector bundle to $\bar{U}_{0}$ (see example 2.6). Then a $\mathbb{C}$-Hodge complex:

$$
A^{n \bullet}(\mathcal{F})=\bigoplus_{U_{0} \leq \ldots \leq U_{n}} j_{\bar{U}_{0} *}\left(W_{0} A_{\bar{U}_{0}}^{\bullet}\left(\log \left(\bar{U}_{0}-U_{0}\right)\right) \otimes V_{\left(U_{0}, \ldots U_{n}\right)}, \ldots\right)
$$

can be constructed on $P \Delta$, where $A_{\bar{U}_{0}}^{\bullet}\left(\log \left(\bar{U}_{0}-U_{0}\right)\right) \otimes V_{\left(U_{0}, \ldots U_{n}\right)}$ is described 2.6 . This produces a natural $\mathbb{C}$-Hodge structure on cohomology with coefficients in $\mathcal{F}$. Similar arguments yield $(\mathbb{C}$-)mixed Hodge structures on the cohomology of arbitrary varieties with constant (or unitary) constructible coefficients. The bookkeeping is a bit more involved in this case. $\mathcal{S} \mathcal{V}$ would be replaced by the category of triples $(X, \mathcal{B}, \bar{X})$ where $\mathcal{B}$ is a stratification of $X$ and $\bar{X}$ a compactification. The objects of $\Delta$ would be triples where $\bar{X}$ is disjoint union of smooth projective varieties, $\mathcal{B}$ a good stratification of the form $\mathcal{B}(E)$ and $(\bar{X}-X) \cup E$ a divisor with normal crossings.

I want to conclude with a few words about how all of this ought to relate to Saito's work on mixed Hodge modules [Sa1, Sa2, Sa3]. I will treat mixed Hodge module theory entirely as black box, and just summarize some of the main properties. For 
a gentle introduction to the subject see $\mathbb{B Z}$. To every variety $X$ over $\mathbb{C}$, Saito constructs the Abelian category $M H M(X)$ of mixed Hodge modules. For a point, $M H M(p t)$ is equivalent to the category of $\mathbb{Q}$-mixed Hodge structures $H$ which are polarizable in the sense that $G r W H$ admits polarizations. There is an additive functor rat : $\operatorname{MHM}(X) \rightarrow D_{c}^{b}(X, \mathbb{Q})$ to the derived category of sheaves of $\mathbb{Q}_{X^{-}}$ modules with bounded constructible cohomology; in fact, rat is an exact and faithful functor to the Abelian subcategory of perverse sheaves [BBD]. When $X$ is smooth, the category of perverse sheaves over $\mathbb{C}$ is exactly the image of the category of regular holonomic $D_{X}$-modules under the De Rham functor $D R$ (by the RiemannHilbert correspondence). In this case, $M H M(X)$ is constructed as a subcategory of the category whose objects are pairs consisting of a filtered regular holonomic $D_{X^{-}}$ module $(M, F)$ and a filtered perverse sheaf over $\mathbb{Q}(K, W)$ such that $D R(M) \cong K \otimes$ $\mathbb{C}$. The functor rat extends to a morphism of triangulated categories denoted by the same symbol rat: $D^{b} M H M(X) \rightarrow D_{c}^{b}(X, \mathbb{Q})$. The basic operations of sheaf theory extend to $D^{b} M H M$. In particular, given a morphism $f: X \rightarrow Y$, there exists morphisms of triangulated categories $\mathbb{R} f_{*}: D^{b} M H M(X) \rightarrow D^{b} M H M(Y)$ and $\mathbb{L} f^{*}: D^{b} M H M(Y) \rightarrow D^{b} M H M(X)$ which are compatible with the corresponding morphisms of $D_{c}^{b}$ (I have added the " $\mathbb{R}$ " and "L" for consistency; Saito leaves these out by convention). These facts together imply that $H^{i}(X, \operatorname{rat}(M))$ is assigned a mixed Hodge structure for each $M \in D^{b} M H M(X)$.

Given a constant constructible sheaf $\mathcal{F}$ on $X$, I expect that there should be a natural choice of $M \in D^{b} M H M(X)$ with $\operatorname{rat}(M)=\mathcal{F} \otimes \mathbb{Q}$ such that $H^{i}(X, M)$ gives the same $\mathbb{Q}$-mixed Hodge structure as above. When $\mathcal{F}=A_{X}$ is constant, the obvious choice for $M$ is $\mathbb{L} f^{*}(A \otimes \mathbb{Q}(0))$, and when $X$ is smooth the mixed Hodge structures on $H^{i}(X, A)$ do coincide by [Sa2, 3.3]. I have a candidate for $M$ for general $\mathcal{F}$, but I don't yet have a proof that it works. The main problem is that the derived category is the wrong place for doing descent, a more "rigid" description of $M$ is needed first. Mixed Hodge modules do not work on simplicial schemes for these reasons; some of these issues are touched upon in a recent preprint of Saito Sa4, but it appears that more work is needed to resolve them.

\section{REFERENCES}

[A] D. Arapura, Geometry of cohomology support loci for local systems I, J. Alg. Geom. 6 (1997)

[SGA4] M. Artin, A. Grothendieck, B. Saint-Donat, J. Verdier, Cohomologie de Topos et cohomologie étale de schémas, Lect. Notes in Math 269, 270, 305, (1972-1973) Springer-Verlag

[B] A. Beilinson, Notes on absolute Hodge cohomology, AMS Contemp. Math. vol 55

[BBD] A. Beilinson, J. Bernstein, P. Deligne, Faisceaux pervers, Astérisque 100 (1982)

[BZ] J-L, Brylinski, S. Zucker, An overview of recent advances in Hodge theory, Several Complex Variables VI, Springer-Verlag (1990)

[Bu] J. I. Burgos, $A C^{\infty} \log$ complex, Compositio Math 92 (1994)

[D1] P. Deligne, Equations différentielles à points singuliers réguliers, Lect. Notes in Math. 163, Springer-Verlag ( 1969)

[D2] P. Deligne, Théorie de Hodge II, III, Publ. IHES 40(1972), 44 (1974)

[D3] P. Deligne, Un théorème de finitude pour la monodromie, Discrete groups in geometry and analysis, Birkhauser (1987)

[E] F. El Zein, Mixed Hodge structures, Trans. of AMS 275 (1983)

[EV] H. Esnault, E. Viehweg, with appendix by K. Timmerscheidt Logarithmic de Rham complexes and vanishing theorems, Inv. Math. 86 (1986)

[GS] H. Gillet, C. Soulé, Descent, motives and K-theory, Crelles, J. 478 (1996)

[G] R. Godement, Théorie des faisceaux Hermann (1958) 
[GL] M. Green, R. Lazarsfeld, Deformation theory, generic vanishing theorems,... Inv. Math. 90 (1987)

[GS] P. Griffiths, W. Schmid, Recent developments in Hodge theory, Discrete subgroups of Lie groups, Tata Inst. (1975)

[GNPP] F. Guillén, V. Navarro Aznar, P. Pascual-Gainza, P. Puerta, Hyperrésolutions cubiques et descente cohomologique Lect. Notes in Math. 1335, Springer-Verlag (1988)

[GN] F. Guillén, V. Navarro Aznar, Un critère d'extension d'un fonctor défini sur les schémas lisses, alg-geom preprint 9505008 (1995)

[Ha] R. Hain, The de Rham homotopy theory of complex algebraic varieties I, II, K-theory 1 (1987),

[Hi] H. Hironaka, Resolution of singularities of an algebraic variety of characteristic zero, Ann. of Math. 79 (1964)

[dJ] J. de Jong, Smoothness, semistability and alterations, Publ. IHES 83 (1996)

[L] B. Lasell, On complex local systems and morphisms of varieties, Comp. Math. 98 (1995)

[M] S. Maclane, Categories for the working mathematician, Springer-Verlag (1971)

[N] V. Navarro Aznar, Sur la théorie de Hodge-Deligne, Inv. Math. 90 (1987)

[Sa1] M. Saito, Modules de Hodge polarisables, Publ. RIMS 24 (1988)

[Sa2] M. Saito, Mixed Hodge modules and admissible variations, CR Acad. 309 (1989)

[Sa3] M. Saito, Mixed Hodge modules, Publ. RIMS 26 (1990)

[Sa4] M. Saito, Mixed Hodge complexes on algebraic varieties, alg-geom preprint 9906088 (1999)

[S] C. Simpson, Higgs bundles and local systems, Publ. IHES 75 (1992)

[St] J. Steenbrink, Limits of Hodge structures, Inv. Math. 31 (1976)

[V] J. L. Verdier, Des Catégories dérivées des catégories abélinnes, Astérisque 239 (1996)

[Z] S. Zucker, Hodge theory with degenerating coefficients, Ann. of Math. 109 (1979)

Department of Mathematics, Purdue University, West Lafayette IN 47907 